\title{
Conjonctions de subordination de temps tant que/aussi longtemps que, jusqu'à ce que, dok : analyse contrastive français-croate
}

\author{
Marija Spajić \\ Maja Pivčević \\ Université de Zadar \\ mspajic@unizd.hr \\ mpivcevic@unizd.hr
}

Cette étude se propose de comparer la distribution et l'utilisation de certaines conjonctions de subordination temporelles en français et en croate (français : tant quel aussi longtemps que, jusqu'à ce que; croate : dok).

Tout en étant universelle, la catégorie grammaticale du temps donne lieu à des conceptualisations langagières tout à fait comparables mais différentes.

Notre expérience linguistique du français et du croate et notamment l'expérience de l'enseignement du français aux croatophones nous ont permis de remarquer que les différences dans leurs conceptualisations respectives suscitent certaines divergences entre les deux systèmes, et sont par conséquent susceptibles d'entraîner des difficultés dans l'apprentissage du français par les croatophones.

Le but de cette étude est de mettre en évidence quelques différences dans l'utilisation de ces conjonctions de subordination temporelles françaises et leurs équivalents croates en tenant compte des temps, modes et aspects ainsi que des rapports chronologiques entre le procès de la proposition principale et celui de la subordonnée introduite par la conjonction temporelle.

Nos observations sur la sémantique de la temporalité s'appuieront sur des exemples puisés à des grammaires et à un corpus parallèle (de textes littéraires français et leurs traductions croates et inversement).

Mots clés : tant que, jusqu'à ce que, propositions terminatives, français, croate

\section{Introduction}

La conjonction de subordination de temps est une unité linguistique qui permet d'identifier une phrase complexe exprimant d'une manière explicite le rapport chronologique entre les procès de ses propositions. De ce fait, les phrases complexes de temps se prêtent à être considérées comme le noyau grammatical de la catégorie sémantique de temporalité, qui, elle, recouvre nombre d'autres moyens exprimant la temporalité implicitement ou bien à l'aide de différentes unités lexicales (cf. Badurina 2013 : 76). 
La subordonnée introduite par une conjonction de temps se rapporte à la proposition principale comme un complément circonstanciel, établissant avec cette dernière, sur le plan sémantique, un rapport chronologique de simultanéité, d'antériorité ou de postériorité. Ces trois rapports servent souvent de repère de description et/ou de classification pour les subordonnées de temps. Silić et Pranjković en isolent, pourtant, les propositions terminatives, à savoir celles qui marquent la limite d'un procès (GHGV : 337-339), et c'est à ce sous-groupe qu'appartiennent les propositions introduites par tant quelaussi longtemps que, jusqu'à ce que et leurs équivalents croates. En comparant le fonctionnement de cette partie des deux systèmes, on remarque des différences dans les champs couverts par telle ou telle conjonction : à trois conjonctions françaises n'en équivaut qu'une seule en croate (dok). L'analyse contrastive de ce type de phrases nous permettra de comprendre comment ces deux systèmes conceptualisent et formalisent les mêmes traits sémantiques, et de montrer où et comment ils divergent. Pour mettre en valeur les divergences, nous analyserons d'abord des exemples illustratifs tirés d'une grammaire didactique française et leurs équivalents croates, en tenant compte non seulement des conjonctions mais aussi des temps et des modes. L'analyse nous permettra de poser des hypothèses sur les équivalences entre les deux systèmes, que nous testerons par la suite sur les exemples puisés dans un corpus parallèle de textes littéraires.

\section{Tant que/aussi longtemps que, jusqu'à ce que et leur équivalent croate}

Si on consulte les grammaires françaises de référence (BU1, BU2, GMF, GFCM), on constate que dans tous ces ouvrages tant que est cité parmi les conjonctions exprimant la simultanéité, tandis que jusqu'à ce que figure parmi celles exprimant l'antériorité ou la postériorité, en fonction du point de vue depuis lequel on envisage les relations dans une phrase. ${ }^{1}$ Puisque $1^{\prime}$ on n'y traite pas de l'emploi de tant que qui serait pertinent pour notre analyse, nous avons eu recours à un ouvrage didactique.

Dans La grammaire expliquée du français, niveau intermédiaire (GEF : 277-285) les propositions temporelles sont réparties en trois groupes, en fonction du rapport qu'elles établissent avec le procès exprimé par le verbe de la principale, à savoir un rapport de simultanéité, d'antériorité ou de postériorité. Tant que et son synonyme sémantiquement plus transparent aussi longtemps que y sont classés parmi les conjonctions indiquant la simultanéité. Selon cet ouvrage, « ces deux conjonctions synonymes montrent que deux actions se déroulent exactement dans le même moment du temps, que ces deux actions ont exactement la même durée » (GEF : 281) :

1 Par ailleurs, jusqu'au XVI ème siècle, jusqu'à ce que est couramment suivi de l'indicatif (cf. BU1 : 1163), ce qui est possible encore aujourd'hui si l'on veut souligner la réalité du fait (cf. BU2 : 1487), alors que tant que, suivi du subjonctif, est employé dans le sens de jusqu'à ce que (cf. BU1 : 890 ; GFCM : 584). Cela nous suggère une proximité sémantique de ces deux conjonctions du point de vue diachronique. 
(1) Tant qu'il travaille, mon ami se sent heureux.

En revanche, à la forme négative, les emplois bifurquent, et sont décrits de la manière suivante :

a) «Quand le verbe de la proposition subordonnée exprime une action continue, un état qui se prolonge, on garde les mêmes temps verbaux dans les deux propositions »:

(2) Tant que tu ne parles pas, tant que tu ne fais pas de bruit, tu peux rester là.

(2) est analogue à (1), à cette différence près qu'ici le verbe de la subordonnée est à la forme négative et que cette négation ne désigne donc pas un procès mais l'absence de ce procès (cf. Mladenović/Samardžija 2019 : 62-63) - dans les deux cas, les procès ou les absences du procès ont exactement la même durée.

b) Cependant, « quand le verbe exprime une action accomplie à un moment donné, un résultat, on constate une différence de temps dans les deux propositions »(GEF : 281) :

(3) Tant qu'elle n'était pas entrée en scène, l'actrice était morte de trac (= l'actrice était morte de trac jusqu'à ce qu'elle soit entrée en scène).

Plus précisément, la négation dans la subordonnée désigne ici l'absence du résultat de ce procès (cf. Mladenović/Samardžija 2019 : 63), et la description citée conviendrait mieux à la transformation explicative dont est accompagné (3).

C'est justement cette transformation qui nous est particulièrement intéressante : y apparaît jusqu'à ce que, pourtant classée parmi les conjonctions indiquant la postériorité. Selon l'ouvrage en question, « cette conjonction montre $q^{\prime} u^{\prime}$ ne action ${ }^{2}$ se poursuit sans discontinuer jusqu'à un point limite » (GEF : 284) :

(4) Je reste dehors [...] jusqu'à ce qu'il revienne.

Jusqu'à ce que apparaissant dans la transformation explicative de (3), il en suivrait que l'on peut, pour (4), procéder de façon inverse :

(4) (= Je reste dehors tant qu'il n'est pas revenu).

Cette transformation peut amener à se demander si tant que pourrait également introduire la postériorité du contenu de la subordonnée, mais la subordonnée étant négative, nous prenons ce fait pour partie intégrante de son contenu, ce qui nous permet d'interpréter la subordonnée (désignant l'absence du résultat du procès) comme simultanée à la principale, même si elle implique, bien évidemment, un point limite où se produit ce résultat (le retour en l'occurrence, postérieur au contenu de la principale).

Observons maintenant les équivalents croates de nos exemples :

(1') Dok radi, moj se prijatelj osjeća sretnim.

(2') Dok ne govoriš, dok ne bučiš, možeš ostati ovdje.

2 C'est-à-dire le procès de la principale. 
(3') Dok nije stupila na scenu, glumica je umirala od treme (= glumica je umirala od treme dok nije stupila na scenu).

(4') Ostajem vani dok se on ne vrati (= ostajem vani dok se on ne vrati).

Dans tous les équivalents croates de tous les exemples avec tant quelaussi longtemps que et jusqu'à ce que apparaît dok, ce qui met en valeur l'ambiguïté, c'est-à-dire la polysémie (cf. Nazalević Čučević 2016 : 340) de cette conjonction et explique la difficulté que peuvent rencontrer les croatophones dans l'acquisition de cette partie du système français. ${ }^{3}$ L'emploi qui nous intéresse le plus est (3), justement celui qui permet la transformation avec jusqu'à ce que (et vice versa) car les équivalents montrent bien que cette transformation n'entraîne aucun changement nécessaire en croate.

\section{Temps, modes et aspects}

Les temps, modes et aspects apportent, eux-aussi, en concurrence avec les conjonctions, des précisions au sens et au rapport chronologique entre les procès des deux propositions.

Dans (1) et (2), le rapport chronologique entre les deux procès continus ayant exactement la même durée est traduit par les deux verbes au même temps de l'indicatif. Le fait que les propositions soient à la forme affirmative ou négative $n^{\prime} y$ change rien. Dans $\left(1^{\prime}\right)$ et $\left(2^{\prime}\right)$, ce rapport est traduit par l'aspect imperfectif des deux verbes.

En revanche, dans (3), celui qui permet la transformation avec jusqu'à ce que, le verbe introduit par tant que est à la forme composée du temps de l'indicatif du verbe de la principale, et il y a négation, alors que dans la transformation avec jusqu'à ce que, le verbe de la subordonnée est au subjonctif affirmatif. En croate, qui n'offre qu'un seul équivalent de ces deux phrases ${ }^{4}$, le verbe de la subordonnée est d'aspect perfectif et il y a négation ${ }^{5}$, tandis que le verbe de la principale reste imperfectif.

3 La polysémie de dok ne se réduit pas aux trois emplois dont il est question ici, mais apparaît également dans les équivalents des propositions françaises avec pendant que, tandis quelalors que, le temps que, avant que. En outre, dans les trois emplois en question, dok est généralement remplaçable par les variantes sve dok, dokle, dok(le) god, tako dugo dok, sve dotle dok, koliko god dugo qui apportent, certes, des nuances sémantiques qui mériteraient une étude approfondie, mais elles ne sont pas pertinentes pour notre propos.

4 Si on transforme (3) au présent (Tant qu'elle n'est pas entrée en scène, l'actrice est morte de trac), l'équivalent en est soit dok nije stupila na scenu, soit dok ne stupi na scenu, glumica umire od treme (cf. Mladenović/Samardžija 2019 : 67).

5 La négation en croate est particulièrement intéressante car la question si elle est, et quand elle est explétive ou non reste ouverte (cf. GHGV : 338-339 ; Nazalević Čučević 2016 ; Mladenović/Samardžija 2019 : 67). Il est évident, en revanche, que sa grande fréquence dans les propositions de ce type pose problème aux apprenants croatophones en les entraînant à utiliser le ne explétif avec jusqu'à ce que, ce qui n'est pas de règle. 


\section{Propositions terminatives}

Dans leur ouvrage Gramatika hrvatskog jezika za gimnazije i visoka učilišta, dans lequel les propositions temporelles sont également réparties selon le rapport chronologique qu'elles établissent avec le procès de la principale, Silić et Pranjković isolent un sous-groupe qui est, à notre connaissance, absent des descriptions françaises homologues : les propositions temporelles dites terminatives (cf. GHGV : 337-339). Selon les auteurs, de telles propositions sont susceptibles de marquer soit le début soit le terme, c'est-à-dire la limite d'un procès. Les propositions sur lesquelles nous portons intérêt en font partie et on les retrouve parmi celles qui mettent au premier plan le terme d'un procès : elles soulignent soit que les procès des deux propositions ont exactement la même durée (5) soit que le procès de la subordonnée commence à se dérouler au moment où le procès de la principale ne se déroule plus $(6)^{6}$ :

(5) Ovdje ostajemo dokle hoćemo.

(5') Nous restons ici tant que nous voulons ${ }^{7}$.

(6) Radit ću sve dotle dok sve ne napravim.

Conformément à ce que nous a montré (3) ci-dessus, le type (6) est celui pour lequel le français dispose de deux équivalents, soit avec tant quelaussi longtemps que soit avec jusqu'à ce que :

(6') Je travaillerai tant quelaussi longtemps que je n'aurai pas tout fait.

Je travaillerai jusqu'à ce que j'aie tout fait/fasse tout.

De ce fait, on peut se poser la question sur la différence sémantique qu'il y aurait entre ces deux équivalents : selon Mladenović et Samardžija (2019: 66), la raison pour laquelle tant que et jusqu'à ce que alternent à condition que tant que introduise un contenu négatif (ou vice versa) réside en cela que «tant que vise non pas ce qui se passe à partir d'un moment dans l'avenir, mais ce qui se passe (ou ne se passe pas) tout le temps avant ce moment ». En revanche, jusqu'à ce que « vise surtout la réalisation du procès visé ».

\section{Hypothèses et corpus parallèle}

L'analyse nous a permis de poser les hypothèses suivantes :

a) L'équivalent de la proposition introduite par tant quelaussi longtemps que + le verbe au même temps que celui de la principale, peu importe s'il y a négation ou pas, est la proposition introduite par $d o k+$ verbe d'aspect imperfectif.

6 C'est à dire, conformément à ce qu'on a dit ci-dessus, la subordonnée étant négative, le procès de la principale est simultané à l'absence du résultat du procès de la subordonnée.

7 On accompagne ici les exemples croates de leurs équivalents français. 
b) Il n'y a qu'un seul équivalent croate de la proposition avec jusqu'à ce que + subjonctif affirmatif et de celle avec tant quelaussi longtemps + indicatif négatif à la forme composée du temps du verbe de la principale : proposition introduite par $d o k+$ verbe d'aspect perfectif, le plus souvent négatif.

Pour tester nos hypothèses, on a puisé des exemples dans les textes En finir avec Eddy Bellegueule, Je m'en vais, Le Petit Prince, Le Roi des Aulnes, Vernon Subutex 1, Vernon Subutex 3 et Messieurs les Glembay.

Pour ce qui est de l'hypothèse 1, des 24 phrases de ce type, 22 la confirment parfaitement; en voilà une à titre d'exemple :

(7) Alors ils écoutaient les Cramps, le Gun Club et MC5 en buvant de la bière, tant que Bertrand la supportait encore. (VSF : 20)

(7') Pa su onda, pijući pivo, dok ga je Bertrand još podnosio, slušali The Cramps, The Gun Club i MC5. (VSC : 14)

L'hypothèse 2 est confirmée par 19 des 20 phrases de ce type ${ }^{8} ; 3$ en sont avec tant quelaussi longtemps, 17 avec jusqu'à ce que ; le verbe croate est négatif dans 19. Un exemple pour chaque conjonction :

(8) Tant qu'elle n'avait pas donné son avis, celui des autres - les applaudissements d'un Zénith-n'était pas validé. (VSF : 134)

(8') Sve dok ona ne bi dala svoj sud, svi drugi, svi aplauzi jednog Zénitha, nisu vrijedili ništa. (VSC : 89)

(9) La première nuit de sa vie sans Jean-No, il avait marché jusqu'à ce que les plantes des pieds le brûlent, puis il avait continué. (VSF : 24)

(9') Prve noći u životu bez Jean-Noa, hodao je dok ga tabani nisu počeli peći, a onda je nastavio. (VSC : 17)

\section{Conclusion}

Nous n'avons qu'effleuré les traits saillants d'une problématique qui exigerait, bien évidemment, une analyse beaucoup plus détaillée, incluant notamment l'aspect lexical et le sémantisme des verbes, ainsi que la position de la subordonnée par rapport à la principale, la question de la négation, explétive ou non, la distribution et les nuances sémantiques des variantes sve dok/dokle/dokle god/dotle dok/tako dugo dok, une enquête avec des locuteurs confirmés et non confirmés, les équivalents des conjonctions en question en d'autres langues, etc. Néanmoins, nous pouvons conclure de tout ce qui vient d'être observé que jusqu'à ce que et tant que sont spécialisés dans le sens de la terminativité alors que le croate, dok

8 Le cadre de cette étude ne nous permettant pas d'interpréter les écarts, nous nous contenterons de dire qu'ils ne semblent être que des variantes qui ne contestent pas notre propos. 
couvrant un champ beaucoup plus large, l'exprime en combinant la conjonction et le jeu des verbes. En outre, puisque pour les propositions avec jusqu'à ce que + subjonctif affirmatif et avec tant quelaussi longtemps + indicatif négatif à la forme composée du temps du verbe de la principale le croate semble ne disposer que d'un seul équivalent (dok + verbe d'aspect perfectif), nous concluons que, du point de vue français, non seulement dok est polysémique, mais la proposition entière de ce type aussi.

\section{Bibliographie}

Badurina, Lada (2013). Vremenski odnosi na razini složene rečenice i teksta, in : Vrijeme u jeziku; Nulti stupanj pisma: zbornik radova 41. seminara Zagrebačke slavističke škole [éd. Tatjana Pišković/Tvrtko Vuković], Zagreb : Filozofski fakultet, Zagrebačka slavistička škola, Hrvatski seminar za slaviste, pp. 75-97.

Grevisse, Maurice (1975). Le bon usage, Dixième édition, Gembloux : Editions J. Duculot. (BU1)

Grevisse, Maurice / Goosse, André (2008). Le bon usage, 14e édition, Bruxelles : De Boeck \& Larcier. (BU2)

Mladenović, Brigitte / Samardžija, Tatjana (2019). Le bon mot, Prevođenje tekstova sa srpskog na francuski sa gramatičkim objašnjenjima, Beograd : Filološki fakultet Univerziteta u Beogradu.

Nazalević Čučević, Iva (2016). Negacija u vremenskim rečenicama s veznikom dok u hrvatskome jeziku, in : Romanoslavica, vol. LII, nr. 2, pp. 331-341.

Poisson-Quinton, Sylvie / Mimran, Reine / Mahéo-Le Coadic, Michelle (2004). La grammaire expliquée du français, niveau intermédiaire, Paris : CLE International. (GEF)

Riegel, Martin / Pellat, Jean-Christophe / Rioul, René (2004). Grammaire méthodique $d u$ français, Paris : Presses Universitaires de France. (GMF)

Silić, Josip / Pranjković, Ivo (2007). Gramatika hrvatskoga jezika za gimnazije i visoka učilišta, Zagreb : Školska knjiga. (GHGV)

Wagner, René-Louis / Pinchon, Jacqueline (1985), Grammaire du français classique et moderne, Paris : Librairie Hachette. (GFCM)

\section{Corpus}

Despentes, Virginie (2015). Vernon Subutex 1, Paris : Grasset. (VSF)

Despentes, Virginie (2016). Vernon Suboteks 1 [trad. Ita Kovač], Zagreb : Naklada OceanMore. (VSC)

Despentes, Virginie (2017). Vernon Subutex 3, Paris : Grasset.

Despentes, Virginie (2016). Vernon Suboteks 3 [trad. Ita Kovač], Zagreb : Naklada OceanMore.

Krleža, Miroslav (1929). Gospoda Glembajevi, <http://gimnazija-sb.com/portal/ wp-content/uploads/2015/02/krleza_gospodaglembajevi.pdf > (15/10/2019) 
Krleža, Miroslav (2017). Messieurs les Glembay [trad. Nicolas Raljević], RueilMalmaison : Prozor éditions.

De Saint-Exupéry, Antoine (1999). Le petit prince, Paris : Éditions Gallimard.

De Saint-Exupéry, Antoine (2015). Mali princ [trad. Goran Rukavina], Split : Marjan Šare.

Echenoz, Jean (1999). Je m'en vais, Paris : Éditions de Minuit.

Echenoz, Jean (2001). Ja odlazim [trad. Ita Kovač], Zagreb : Naklada Enigma.

Louis, Édouard (2014). En finir avec Eddy Bellegueule, Paris : Éditions du Seuil.

Louis, Édouard (2019). Raskrstimo s Eddyjem [trad. Ita Kovač], Zagreb : Naklada

OceanMore.

Tournier, Michel (1970). Le Roi des Aulnes, Paris : Gallimard.

Tournier, Michel (2008). Kralj Joha [trad. Bosiljka Brlečić], Zagreb : Lunapark.

\section{Kontrastivna analiza francuskih i hrvatskih vremenskih veznika tant que/aussi longtemps que, jusqu'à ce que, dok}

U radu se uspoređuju distribucija i uporaba nekih vremenskih veznika u francuskom i hrvatskom jeziku (francuski: tant que/aussi longtemps que, jusqu'à ce que; hrvatski: dok).

Služeći se francuskim i hrvatskim te, naročito, podučavajući francuski ovjerenim kroatofonim govornicima osvijestili smo da se razlike u konceptualizacijama u tim dvama jezičnim sustavima očituju u stanovitim divergencijama koje kroatofonim govornicima pri učenju francuskog nesumnjivo mogu uzrokovati poteškoće.

Cilj ovog rada ukazati je na razlike u uporabi navedenih veznika $u$ francuskome i njihovih ekvivalenata u hrvatskome, uzimajući u obzir glagolsko vrijeme, način i vid, kao i odnose istovremenosti ili sukcesivnosti između radnji glavne i zavisne surečenice uvedene vremenskim veznikom.

Opažanja o semantici temporalnih odnosa temelje se na primjerima iz gramatika te iz paralelnog korpusa francuskih književnih tekstova i njihovih prijevoda na hrvatski i obrnuto. Analiza nas je dovela do dviju u velikoj mjeri potvrđenih hipoteza: zavisnoj surečenici s veznikom tant quelaussi longtemps que, u kojoj je radnja u glavnoj surečenici izražena predikatom, zanijekanim ili ne, u istom vremenu kao i u zavisnoj surečenici, hrvatski je ekvivalent surečenica s veznikom dok i predikatom nesvršenog vida (1); zavisnoj surečenici s veznikom jusqu'à ce que i predikatom u potvrdnom obliku konjunktiva te zavisnoj surečenici s veznikom tant quelaussi longtemps que i predikatom u niječnom, složenom obliku indikativa glavne surečenice, jedini je hrvatski ekvivalent surečenica s veznikom dok i predikatom svršenog vida, najčešće u niječnom obliku (2).

Ključne riječi: tant que, jusqu'à ce que, terminativne rečenice, francuski jezik, hrvatski jezik 